\title{
Event Design in Event-Triggered Feedback Control Systems
}

\author{
Xiaofeng Wang and Michael D. Lemmon
}

\begin{abstract}
This paper studies the event design in eventtriggered feedback systems with asymptotic stability. A new event-triggering scheme is presented that may postpone the occurrence of events over previously proposed methods. Our approach pertains to nonlinear state-feedback systems. The resulting event-triggered feedback systems are guaranteed to be asymptotically stable, provided that the continuous systems are stabilizable. We also show that the task periods and deadlines generated by our scheme are bounded strictly away from zero if the continuous systems are input-to-state stable with respect to measurement errors. Simulation results indicate that our eventtriggered scheme has a much larger average period compared with the prior work. Moreover, our scheme also appears to be robust to task delays.
\end{abstract}

\section{INTRODUCTION}

Sampled-data systems sample continuous signals and use computers to make control decisions based on the sampled data. A great challenge of implementing sampled-data systems is to determine the sample periods such that the resulting systems achieve a desired level of performance.

Traditional approaches are based on periodic task models, in which consecutive invocations of a task are released in a periodic manner. Lyapunov techniques were used in Zheng et al. [1] for a class of nonlinear sampled-data systems. Following that, Nesic et al. [2] used input-to-state stability (ISS) techniques to bound the inter-sample behavior of nonlinear systems. $\mathcal{L}_{p}$ stability of such sampled-data systems was considered in [3]. For networked control systems, the maximum admissible time interval (MATI) was introduced by Walsh et al [4]. Further work was done in [5], [6].

As we mentioned above, the preceding approaches are all based on periodic task models. Such models may be undesirable in many situations due to their conservativeness. Under periodic task models, the selection of sample periods is done before the system is deployed. One therefore has to ensure adequate behavior over a wide range of uncertainties. As a result, these selected periods may be shorter than necessary, which results in significant over-provisioning of the real-time system hardware. This over-provisioning may negatively impact the scheduling of other tasks on the same processing system. In these applications it may be better to consider alternatives to periodic task models that can more effectively balance the real-time system's computational cost against the control system's performance.

In recent years, sporadic task models have been considered for real-time control. A hardware realization of such

Both authors are with the department of electrical engineering, University of Notre Dame, Notre Dame, IN 46556; e-mail: xwang13,lemmon@nd.edu. The authors gratefully acknowledge the partial financial support of the National Science Foundation (NSF-CNS-0720457) models is called event-triggering. Under event-triggering the system states are sampled when some error signal exceeds a given threshold [7], [8], [9], [10]. Event-triggering requires a hardware event detector that may be implemented using custom analog integrated circuits (ASIC's) or floating point gate array (FPGA) processors. A software realization of these sporadic task models is called self-triggering. Under selftriggering the next task release time is predicted by the processing computer based on the current sampled state [11], [12], [13]. This software approach may be appropriate when the hardware implementation is unacceptable.

Both realizations of sporadic task models have the ability to dynamically adjust the task periods to variations in the system state. This "on-line" property enables event/selftriggering to generate longer task periods than periodic task models [13]. One thing worth mentioning is that, provided the cost associated with using ASIC/FPGA hardware is acceptable, event-triggering has a lower computational cost than self-triggering and usually generates longer sample periods since self-triggering periods are usually conservative estimates of the periods generated by event-triggering.

This paper studies the event design for event-triggered feedback systems with asymptotic stability. In all of the prior work considering stability of event/self-triggered feedback systems [9], [10], [12], [13], the same Lyapunov function $V$ is shared by the continuous closed-loop system and the sampled-data system. Meanwhile, $V$ is required to be monotone decreasing in the sampled-data system as it is in the continuous system. This is not necessary. As noted in earlier work on switched system stability, we can still guarantee asymptotic stability as long as an appropriate subsequence of $V$ is monotone decreasing [14]. $V$ need not be decreasing all the time. What this means is that we can lengthen the period between events by adopting this less restrictive condition on $V$ in the sampled-data system.

Based on this idea, a new event-triggering scheme is presented. Our approach pertains to nonlinear state-feedback systems. The resulting event-triggered feedback systems are guaranteed to be asymptotically stable, provided that the continuous systems are stabilizable. We also show that the task periods and deadlines generated by our scheme are bounded strictly away from zero if the continuous systems are input-to-state stable with respect to measurement errors. Simulation results indicate that our event-triggered scheme has a much larger average period than the event-triggered scheme proposed in [9], the self-triggered scheme in [13], and the MATI in [6]. Moreover, our scheme also appears to be robust to task delays.

This paper is organized as follows. In section II the prob- 
lem is formulated. Section III presents the event-triggering scheme. Event-triggered feedback systems with non-zero delays are considered in section IV. Simulation results are presented in section V. Finally, conclusions are stated in section VI.

\section{Problem Formulation}

Consider a nonlinear system with the state equation

$$
\begin{aligned}
& \dot{x}(t)=f(x(t), u(t)) \\
& u(t)=\gamma(x(t)) \\
& x(0)=x_{0}
\end{aligned}
$$

where $x:[0, \infty) \rightarrow \mathbb{R}^{n}$ is the state trajectory, $x_{0} \in \mathbb{R}^{n}$ is the non-zero initial state, and $u:[0, \infty) \rightarrow \mathbb{R}^{m}$ is a control input. In the above equation, $f: \mathbb{R}^{n} \times \mathbb{R}^{m} \rightarrow \mathbb{R}^{n}$ and $\gamma: \mathbb{R}^{n} \rightarrow \mathbb{R}^{m}$ are locally Lipschitz functions.

Assumption 2.1: For the continuous closed-loop system in equation 1 , assume that there exist positive constants $L, \underline{\alpha}, \bar{\alpha}, \underline{\beta}, \bar{\beta}, L_{1} \in \mathbb{R}^{+}$, a positive definite, $\mathbb{C}^{1}$ function $V$ : $\mathbb{R}^{n} \rightarrow \mathbb{R}^{+}$, and two class $\mathcal{K}$ functions $\alpha_{1}, \alpha_{2}: \mathbb{R}^{+} \rightarrow \mathbb{R}^{+}$ such that

$$
\begin{aligned}
& \|f(x, \gamma(x+e))\| \leq L\|x\|+L\|e\| \\
& \alpha_{1}(\|x\|) \leq V(x) \leq \alpha_{2}(\|x\|) \\
& -\underline{\alpha} V(x)-\underline{\beta}\|e\| \\
& \frac{\partial V(x)}{\partial x} f(x, \gamma(x+e)) \leq-\bar{\alpha} V(x)+\bar{\beta}\|e\| \\
& \alpha_{1}^{-1}(\|x\|) \leq L_{1}\|x\|
\end{aligned}
$$

hold for all $x, e$ in a compact set.

Remark 2.1: Equation 4 implies the continuous system $\dot{x}=f(x, \gamma(x+e))$ is ISS with respect to $e$. It also suggests that the continuous closed-loop system in equation 1 is exponentially stable. Further discussion on this assumption can be seen in [9].

Remark 2.2: For a linear time invariant system

$$
\dot{x}(t)=A x(t)+B u, \quad u=K x, \quad x(0)=x_{0},
$$

assumption 2.1 is satisfied as long as the system defined in equation 6 is asymptotically stable. Assume $W=x^{T} P x$ is Lyapunov function of this LTI system. Then $V=\sqrt{W}=$ $\sqrt{x^{T} P x}$ is also a Lyapunov function. Let $Q=-P(A+$ $B K)-(A+B K)^{T} P>0$. So we have

$$
\begin{aligned}
& L=\max \{\|A+B K\|,\|B K\|\}, \\
& \underline{\alpha}=\frac{1}{2} \sigma_{\max }\left(P^{-1} Q\right), \quad \bar{\alpha}=\frac{1}{2} \sigma_{\min }\left(P^{-1} Q\right), \\
& \underline{\beta}=\bar{\beta}=\|\sqrt{P} B K\|, \quad L_{1}=\frac{1}{\sqrt{\sigma_{\min }(P)}} .
\end{aligned}
$$

where $\sigma_{\min }(P)$ denote the minimum singular value of $P$.

A sampled-data implementation of the closed-loop system in equation 1 is

$$
\begin{aligned}
& \dot{x}(t)=f(x(t), u(t)) \\
& u(t)=\gamma\left(x\left(r_{k}\right)\right) \\
& x(0)=x_{0}
\end{aligned}
$$

for $t \in\left[f_{k}, f_{k+1}\right)$ and all $k=0, \ldots, \infty$, where $r_{k}$ denotes the time when the $k$ th invocation of a control task (also called "job") is released for execution on the computer and $f_{k}$ denotes the time when the $k$ th job has finished executing. We assume that the system state is also sampled at $r_{k}$. To simplify the notation, let $T_{k}=r_{k+1}-r_{k}$ denote the $k$ th sample period, $D_{k}=f_{k}-r_{k}$ denote the $k$ th task delay. We also define $e_{k}: \mathbb{R}^{+} \rightarrow \mathbb{R}$ as $e_{k}(t)=x(t)-x\left(r_{k}\right)$ over $t \in\left[r_{k}, f_{k+1}\right)$ for $k \in \mathbb{Z}^{+}$, which is the measurement error.

This paper considers how to reduce the usage of the computational resource subject to the guarantee that the sampled-data system is asymptotically stable. The main idea is to use the violation of events to trigger the release of the task so that (1) the event-triggered system is asymptotically stable and (2) the sample periods can be as long as possible. In the following sections, we will present our approach to designing such events and discuss the robustness of the resulting event-triggered feedback system to external disturbances and delays.

\section{Event-TRIGGERED FEEDBACK Systems}

This section introduces the approach to designing the events that are used to trigger the tasks' release such that the resulting event-triggered feedback system has asymptotic stability. To show asymptotic stability of such a system, we only need to show the existence of a piecewise continuous function $h: \mathbb{R}^{+} \times \mathbb{R}^{n} \rightarrow \mathbb{R}^{+}$such that

$$
\begin{aligned}
& h\left(t \mid x_{0}\right) \geq V\left(x\left(t \mid x_{0}\right)\right), \text { for all } t \in \mathbb{R}^{+} \\
& \lim _{t \rightarrow \infty} h\left(t \mid x_{0}\right)=0,
\end{aligned}
$$

hold, where $x$ is the state trajectories of the resulting eventtriggered feedback system. Since $x$ is also a function of $t$, we use $V(t)$ to denote $V\left(x\left(t \mid x_{0}\right)\right)$.

The results in this section focus on sampled-data systems where $r_{k}=f_{k}$ holds for all $k \in \mathbb{Z}^{+}$. We will propose an event-triggering scheme and show that the resulting system is asymptotically stable and the sampling period $T_{k}$ is bounded from below by a positive constant. To show this, we first introduce a lemma, which will be used in the later proofs.

Lemma 3.1: For two $\mathbb{C}^{1}$ functions $p, q: \mathbb{R}^{+} \rightarrow \mathbb{R}$, assume $\xi \in \mathbb{R}^{+}$is the smallest positive solution to $p(t)=q(t)$. The following statements hold:

1) If $p(0)=q(0), \dot{q}(0)<\dot{p}(0)$, and $t^{*}>0$ satisfies $p\left(t^{*}\right) \leq q\left(t^{*}\right)$, then $t^{*} \geq \xi$

2) If $p(0)>q(0)$ and $t^{*}>0$ satisfies $p\left(t^{*}\right) \leq q\left(t^{*}\right)$, then $t^{*} \geq \xi$

3) If $p(0)=q(0)$ and $\dot{q}(0)<\dot{p}(0)$, then $p(t) \geq q(t)$ for all $t \in[0, \xi)$

4) If $p(0)>q(0)$, then $p(t) \geq q(t)$ for all $t \in[0, \xi)$.

Proof: It can be easily shown taking advantage of the continuity of $p, q, \dot{p}, \dot{q}$.

Theorem 3.2: For the sampled-data system in equation 8, let assumption 2.1 hold and $D_{k}=0$ for all $k \in \mathbb{Z}^{+}$. If $r_{0}=0$ and the $k+1$ th task release is triggered by the violation of

$$
V(t) \leq-\delta \bar{\alpha} V\left(r_{k}\right)\left(t-r_{k}\right)+V\left(r_{k}\right),
$$

where $\delta \in(0,1)$, then the sampled-data system is asymptotically stable and there exists a positive constant $\xi>0$, such that the sample period, $T_{k}$, satisfies $T_{k} \geq \xi$. 
Proof: We first show that the sampling period $T_{k}$ is bounded from below by a positive constant. By equation 2 ,

$$
\frac{d}{d t}\left\|e_{k}(t)\right\| \leq\|\dot{x}(t)\| \leq 2 L\left\|e_{k}(t)\right\|+L\left\|x\left(r_{k}\right)\right\|
$$

holds for all $t \in\left[r_{k}, r_{k+1}\right)$. Solving this differential inequality with the initial condition $e_{k}\left(r_{k}\right)=0$ yields

$$
\left\|e_{k}(t)\right\| \leq \frac{\left\|x\left(r_{k}\right)\right\|}{2}\left(e^{2 L T_{k}}-1\right)
$$

for all $t \in\left[r_{k}, r_{k+1}\right)$. According to equation 3 and 5 ,

$$
\left\|x\left(r_{k}\right)\right\| \leq \alpha_{1}^{-1}\left(V\left(r_{k}\right)\right) \leq L_{1} V\left(r_{k}\right)
$$

holds. Applying equation 12 and 13 into equation 4 leads to the inequality $\dot{V}(t) \leq-\bar{\alpha} V(t)+\frac{\bar{\beta} L_{1} V\left(r_{k}\right)}{2}\left(e^{2 L T_{k}}-1\right)$ for $t \in\left[r_{k}, r_{k+1}\right)$. Solving this differential inequality with the initial condition $V\left(r_{k}\right)$ provides

$$
\begin{aligned}
V(t) \leq & V\left(r_{k}\right) e^{-\bar{\alpha}\left(t-r_{k}\right)} \\
& -\frac{\bar{\beta} L_{1} V\left(r_{k}\right)}{2 \bar{\alpha}}\left(e^{2 L T_{k}}-1\right)\left(e^{-\bar{\alpha}\left(t-r_{k}\right)}-1\right)
\end{aligned}
$$

for all $t \in\left[r_{k}, r_{k+1}\right)$. Because $r_{k+1}$ is triggered by the violation of equation 11 ,

$$
V\left(r_{k+1}\right)=-\delta \bar{\alpha} V\left(r_{k}\right) T_{k}+V\left(r_{k}\right)
$$

holds. Combining equation 14 and 15 yields

$$
\begin{aligned}
& p\left(T_{k}\right) \triangleq-\bar{\alpha} \delta T_{k}+1 \\
& \leq e^{-\bar{\alpha} T_{k}}-\frac{\bar{\beta} L_{1}}{2 \bar{\alpha}}\left(e^{2 L T_{k}}-1\right)\left(e^{-\bar{\alpha} T_{k}}-1\right) \triangleq q\left(T_{k}\right) .
\end{aligned}
$$

It is obvious that $p(0)=q(0)=1$ and $\dot{q}(0)=-\bar{\alpha}<$ $\dot{p}(0)=-\bar{\alpha} \delta$. Therefore, using lemma 3.1, we conclude

$$
T_{k} \geq \xi
$$

where $\xi$ is the smallest positive solution to the equation $p(t)=q(t)$.

We now show asymptotic stability of the resulting system. First, define the function $h\left(t \mid x_{0}\right)$ as:

$h\left(t \mid x_{0}\right)=-\delta \bar{\alpha} V\left(r_{k}\right)\left(t-r_{k}\right)+V\left(r_{k}\right), \forall t \in\left[r_{k}, r_{k+1}\right)$.

Because $r_{k+1}$ is triggered by the violation of equation 11 ,

$$
V(t) \leq-\delta \bar{\alpha} V\left(r_{k}\right)\left(t-r_{k}\right)+V\left(r_{k}\right)=h\left(t \mid x_{0}\right)
$$

holds for all $t \in\left[r_{k}, r_{k+1}\right), k \in \mathbb{Z}^{+}$.

To show asymptotic stability of the system, we still need to show $\lim _{t \rightarrow \infty} h\left(t \mid x_{0}\right)=0$. By the definition of $h\left(t \mid x_{0}\right)$ in equation 17, we know $h\left(t \mid x_{0}\right)$ is differentiable for $t \in$ $\left(r_{k}, r_{k+1}\right)$. By equation 3 , the derivative of $h\left(t \mid x_{0}\right)$ is

$$
\dot{h}\left(t \mid x_{0}\right)=-\delta \bar{\alpha} V\left(r_{k}\right) \leq-\delta \bar{\alpha} \cdot \alpha_{1}\left(\left\|x\left(r_{k}\right)\right\|\right)
$$

for all $t \in\left(r_{k}, r_{k+1}\right)$, which means $h\left(t \mid x_{0}\right)$ is decreasing over $\left(r_{k}, r_{k+1}\right)$.

Although $h\left(t \mid x_{0}\right)$ may not be differentiable at $t=r_{k}$ for some $k \in \mathbb{Z}^{+}$, it satisfies

$$
\begin{aligned}
& \lim _{t \rightarrow r_{k}^{-}} h\left(t \mid x_{0}\right)=\lim _{t \rightarrow r_{k}^{+}} h\left(t \mid x_{0}\right)=h\left(r_{k} \mid x_{0}\right), \\
& \lim _{t \rightarrow r_{k}^{-}} \dot{h}\left(t \mid x_{0}\right) \leq-\delta \bar{\alpha} \cdot \alpha_{1}\left(\left\|x\left(r_{k-1}\right)\right\|\right), \text { and } \\
& \lim _{t \rightarrow r_{k}^{+}} \dot{h}\left(t \mid x_{0}\right) \leq-\delta \bar{\alpha} \cdot \alpha_{1}\left(\left\|x\left(r_{k}\right)\right\|\right)
\end{aligned}
$$

which means for any $k \in \mathbb{Z}^{+}, h\left(t \mid x_{0}\right)$ is continuous at $t=r_{k}$ and the left-hand and right-hand sided derivatives of $h\left(t \mid x_{0}\right)$ at $t=r_{k}$ are both negative. Since equation 16 holds, $r_{k} \rightarrow$ $\infty$ holds. Combining this with equation 19 and 20 yileds

$$
\lim _{t \rightarrow \infty} h\left(t \mid x_{0}\right)=0 \text {. }
$$

Since equation 18 and 21 are satisfied, we can conclude that the sampled-data system is asymptotically stable.

Remark 3.1: For any $k \in \mathbb{Z}^{+}, r_{k+1}$ is triggered when $V(t)$ intersects the straight line $V(t)=-\delta \bar{\alpha} V\left(r_{k}\right)(t-$ $\left.r_{k}\right)+V\left(r_{k}\right)$. This line serves as the threshold. However, using linear functions of $t$ as the thresholds is not the only choice for the threshold. Nonlinear functions can also be used as long as the sequence $\left\{x\left(r_{k}\right)\right\}_{k=1}^{\infty}$ converges to zero and $r_{k+1}-r_{k}>0$ holds for all $k \in \mathbb{Z}^{+}$.

\section{NON-ZERO DELAYS}

In this section, we consider event-triggered feedback systems with non-zero delays and show the existence of a nonzero deadline for the delays with the guarantee of system stability. The main idea is to use an upper bound of $V\left(f_{k}\right)$ as the starting point of the threshold line. $r_{k+1}$ is still triggered when $V(t)$ intersects the threshold line. To ensure the system stability, we need to properly choose the deadline such that the sequence $\left\{V\left(r_{k}\right)\right\}_{k=1}^{\infty}$ converges to zero. In that way, a piecewise continuous $h\left(t \mid x_{0}\right)$ can be constructed satisfying equation 9 and 10 . This is shown in figure 1 , where the horizontal axis is time, the vertical axis is the energy $V$, the solid curve is the trajectory of $V(t)$, and the dashed lines are the threshold lines.

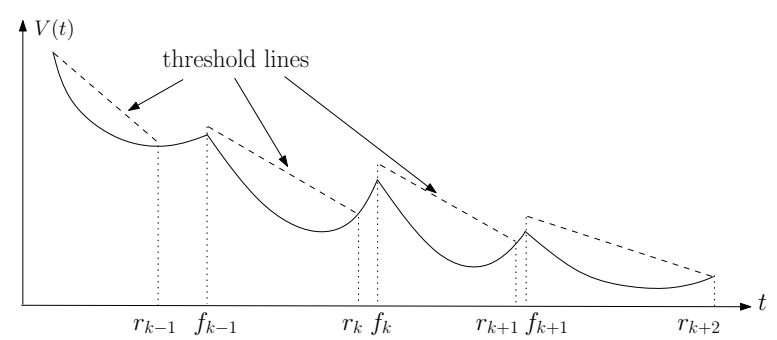

Fig. 1. The trajectory of $V$ and the threshold lines in event-triggered systems with non-zero delays

As we mentioned above, the first step is to find the upper bound for $V(t)$ for $t \in\left[r_{k}, f_{k}\right)$, which is shown in the following lemma.

Lemma 4.1: For the sampled-data system in equation 8, let assumption 2.1 hold. For any $k \in \mathbb{Z}^{+}$, if $V\left(r_{k-1}\right) \leq$ $\lambda V\left(r_{k}\right)$ and the delay $D_{k}$ satisfies $D_{k}<\min \left\{\Delta_{1}, \Delta_{2}\right\}$, where $\lambda \in(1, \infty)$ and $\Delta_{1}, \Delta_{2}$ are the smallest positive solutions to the equation

$$
\begin{gathered}
\frac{\bar{\beta} L_{1}}{\bar{\alpha}}\left[\left(1+\frac{3 \lambda}{2}\right) e^{2 L \Delta_{1}}-\frac{\lambda}{2}\right]\left(1-e^{-\bar{\alpha} \Delta_{1}}\right)=\rho \Delta_{1}, \\
e^{-\underline{\alpha} \Delta_{2}}+\frac{\underline{\beta} L_{1}}{\alpha}(1+\lambda) e^{2 L \Delta_{2}}\left(e^{-\underline{\alpha} \Delta_{2}}-1\right) \\
+\frac{\underline{\beta} L_{1}}{\underline{\alpha}} \frac{\lambda}{2}\left(e^{\frac{\alpha}{2 L} \Delta_{2}}-1\right)\left(e^{-\underline{\alpha} \Delta_{2}}-1\right)=\frac{1}{\lambda},
\end{gathered}
$$


respectively, then $V(t) \leq V\left(r_{k}\right)\left(1+\rho D_{k}\right)$ and $\lambda V\left(f_{k}\right) \geq$ $V\left(r_{k}\right)$ hold for all $t \in\left[r_{k}, f_{k}\right)$, where $\rho \in \mathbb{R}^{+}$is a positive constant satisfying $\rho>\bar{\beta} L_{1}(1+\lambda)$.

Proof: Consider the derivative of $\left\|e_{k-1}(t)\right\|$.

$$
\frac{d}{d t}\left\|e_{k-1}(t)\right\| \leq 2 L\left\|e_{k-1}(t)\right\|+L\left\|x\left(r_{k-1}\right)\right\|
$$

holds for all $t \in\left[r_{k}, f_{k}\right)$. Solving the differential inequality with the initial condition $\left\|e_{k-1}\left(r_{k}\right)\right\|$, we have

$$
\left\|e_{k-1}(t)\right\| \leq\left\|e_{k-1}\left(r_{k}\right)\right\| e^{2 L D_{k}}+\frac{\left\|x\left(r_{k-1}\right)\right\|}{2}\left(e^{2 L D_{k}}-1\right)
$$

for all $t \in\left[r_{k}, f_{k}\right)$. By equation 4 , the inequality

$$
\dot{V}(t) \leq-\bar{\alpha} V(t)+\bar{\beta}\left\|e_{k-1}(t)\right\|
$$

holds for $t \in\left[r_{k}, f_{k}\right)$. Combining this inequality with equation 24 yields

$$
\begin{aligned}
\dot{V}(t) \leq & -\bar{\alpha} V(t)+\bar{\beta}\left\|e_{k-1}\left(r_{k}\right)\right\| e^{2 L D_{k}} \\
& +\bar{\beta} \frac{\left\|x\left(r_{k-1}\right)\right\|}{2}\left(e^{2 L D_{k}}-1\right)
\end{aligned}
$$

for all $t \in\left[r_{k}, f_{k}\right)$. Solving this differential inequality with the initial condition $V\left(r_{k}\right)$ leads to

$$
\begin{aligned}
V(t) \leq & V\left(r_{k}\right) e^{-\bar{\alpha}\left(t-r_{k}\right)} \\
& -\frac{\bar{\beta}}{\bar{\alpha}}\left\|e_{k-1}\left(r_{k}\right)\right\| e^{2 L D_{k}}\left(e^{-\bar{\alpha}\left(t-r_{k}\right)}-1\right) \\
& -\frac{\bar{\beta}}{\bar{\alpha}} \frac{\left\|x\left(r_{k-1}\right)\right\|}{2}\left(e^{2 L D_{k}}-1\right)\left(e^{-\bar{\alpha}\left(t-r_{k}\right)}-1\right)
\end{aligned}
$$

for all $t \in\left[r_{k}, f_{k}\right)$. Using $\|x\| \leq \alpha_{1}^{-1}(V(x)) \leq L_{1} V(x)$ and $V\left(r_{k-1}\right) \leq \lambda V\left(r_{k}\right)$, equation 25 implies

$$
\begin{array}{r}
V(t) \leq V\left(r_{k}\right)- \\
-\frac{\bar{\beta} L_{1}}{\bar{\alpha}}(1+\lambda) V\left(r_{k}\right) e^{2 L D_{k}}\left(e^{-\bar{\alpha} D_{k}}-1\right) \\
-\frac{\bar{\beta} L_{1}}{\bar{\alpha}} \frac{\lambda V\left(r_{k}\right)}{2}\left(e^{2 L D_{k}}-1\right)\left(e^{-\bar{\alpha} D_{k}}-1\right)
\end{array}
$$

for all $t \in\left[r_{k}, f_{k}\right)$. By lemma 3.1 and $D_{k}<\Delta_{1}$, we know

$$
\begin{aligned}
& 1-\frac{\bar{\beta} L_{1}}{\bar{\alpha}}(1+\lambda) e^{2 L D_{k}}\left(e^{-\bar{\alpha} D_{k}}-1\right) \\
& -\frac{\bar{\beta} L_{1}}{\bar{\alpha}} \frac{\lambda}{2}\left(e^{2 L D_{k}}-1\right)\left(e^{-\bar{\alpha} D_{k}}-1\right) \leq 1+\rho D_{k}
\end{aligned}
$$

where $\rho>\bar{\beta} L_{1}(1+\lambda)$. According to equation 26 and 27, it is easy to show that $V(t) \leq V\left(r_{k}\right)\left(1+\rho D_{k}\right)$ holds for all $t \in\left[r_{k}, f_{k}\right)$. Using the similar technique, we can show that if $D_{k}<\Delta_{2}, \lambda V\left(f_{k}\right) \geq V\left(r_{k}\right)$ holds.

Lemma 4.1 shows that $V(t)$ is bounded by a linear function of delays for $t \in\left[r_{k}, f_{k}\right)$ as long as $D_{k}<\Delta_{1}$. Based on this lemma, we can use the point $\left(f_{k}, V\left(r_{k}\right)(1+\rho \Delta)\right)$ as the start of the threshold line with the slope $-\delta \bar{\alpha} V\left(r_{k}\right)$, where $\Delta \leq \Delta_{1}$ is the deadline for the delays. Asymptotic stability of the event-triggered feedback system is guaranteed by theorem 4.3 . The proof of theorem 4.3 requires the following lemma, which shows that the time length between the finishing time, $f_{k}$, and the time instant when $V(t)$ intersects the threshold line is always bounded from below by a positive function of the deadline.

Lemma 4.2: For the sampled-data system in equation 8, let assumption 2.1 hold. If $V\left(r_{k-1}\right) \leq \lambda V\left(r_{k}\right)$ and the delay $D_{k}$ satisfies $D_{k}<\Delta=\min \left\{\Delta_{1}, \Delta_{3}\right\}$, where $\lambda>1, \Delta \in$ $\mathbb{R}^{+}, \Delta_{1}$ is given by equation 22 , and $\Delta_{3} \in \mathbb{R}^{+}$is the smallest positive solution to

$$
\bar{\beta} L_{1} \frac{2+\lambda}{2}\left(e^{2 L \Delta_{3}}-1\right)-\bar{\alpha}\left(1+\rho \Delta_{3}-\delta\right)=0,
$$

then $t_{k}^{*}-f_{k} \geq \xi(\Delta)>0$ holds, where $t_{k}^{*} \geq f_{k}$ is the first time when

$$
V\left(t_{k}^{*}\right)=(1+\rho \Delta) V\left(r_{k}\right)-\delta \bar{\alpha} V\left(r_{k}\right)\left(t_{k}^{*}-f_{k}\right) .
$$

holds after $f_{k}, \rho>\bar{\beta} L_{1}(1+\lambda), \xi(\Delta)$ is the smallest positive solution to

$$
1+\rho \Delta-\delta \bar{\alpha} \tau=(1+\rho \Delta) e^{-\bar{\alpha} \tau}-\frac{g(\tau, \Delta)\left(e^{-\bar{\alpha} \tau}-1\right)}{\bar{\alpha}},
$$

with respect to $\tau$, and $g: \mathbb{R}^{+} \times \mathbb{R}^{+} \rightarrow \mathbb{R}^{+}$is defined by

$$
g(\tau, \Delta)=\bar{\beta} L_{1}\left[\frac{2+\lambda}{2}\left(e^{2 L \Delta}-1\right) e^{2 L \tau}+\frac{1}{2}\left(e^{2 L \tau}-1\right)\right] .
$$

Proof: Consider the derivative of $\left\|e_{k}(t)\right\|$ over the interval $t \in\left[r_{k}, f_{k}\right)$ :

$$
\frac{d}{d t}\left\|e_{k}(t)\right\| \leq 2 L\left\|e_{k}(t)\right\|+L\left\|x\left(r_{k}\right)\right\|+L\left\|e_{k-1}\left(r_{k}\right)\right\| \text {. }
$$

Solving this differential inequality with the initial condition $e_{k}\left(r_{k}\right)=0$, we have

$$
\left\|e_{k}(t)\right\| \leq \frac{\left\|x\left(r_{k}\right)\right\|+\left\|e_{k-1}\left(r_{k}\right)\right\|}{2}\left(e^{2 L D_{k}}-1\right)
$$

for all $t \in\left[r_{k}, f_{k}\right)$. The derivative of $e_{k}(t)$ over $\left[f_{k}, t_{k}^{*}\right)$ satisfies $\frac{d}{d t}\left\|e_{k}(t)\right\| \leq 2 L\left\|e_{k}(t)\right\|+L\left\|x\left(r_{k}\right)\right\|$. Solving this differential inequality with the initial condition given by equation 32 , we have

$$
\left\|e_{k}(t)\right\| \leq \frac{\left\|x\left(r_{k}\right)\right\|+\left\|e_{k-1}\left(r_{k}\right)\right\|}{2}\left(e^{2 L D_{k}}-1\right) e^{2 L\left(t_{k}^{*}-f_{k}\right)}
$$

holds for all $t \in\left[f_{k}, t_{k}^{*}\right)$. By equation 4 and 33 , we have

$$
\begin{aligned}
\dot{V}(t) \leq & -\bar{\alpha} V(t)+\bar{\beta} \frac{\left\|x\left(r_{k}\right)\right\|}{2}\left(e^{2 L\left(t_{k}^{*}-f_{k}\right)}-1\right) \\
& +\bar{\beta} \frac{\left\|x\left(r_{k}\right)\right\|+\left\|e_{k-1}\left(r_{k}\right)\right\|}{2}\left(e^{2 L D_{k}}-1\right) e^{2 L\left(t_{k}^{*}-f_{k}\right)}
\end{aligned}
$$

for all $t \in\left[f_{k}, t_{k}^{*}\right)$. Since $\left\|x\left(r_{k}\right)\right\| \leq \alpha_{1}^{-1}\left(V\left(r_{k}\right)\right) \leq$ $L_{1} V\left(r_{k}\right)$ for all $k \in \mathbb{Z}^{+}$and $V\left(r_{k-1}\right) \leq \lambda V\left(r_{k}\right)$, the inequality above can be further reduced as

$$
\dot{V}(t) \leq-\bar{\alpha} V(t)+g\left(t_{k}^{*}-f_{k}, D_{k}\right) V\left(r_{k}\right),
$$

for all $t \in\left[f_{k}, t_{k}^{*}\right)$. Solving the differential inequality in equation 34 with the initial condition $V\left(f_{k}\right)$ leads to

$$
\begin{aligned}
V\left(t_{k}^{*}\right) \leq & V\left(f_{k}\right) e^{-\bar{\alpha}\left(t_{k}^{*}-f_{k}\right)} \\
& -\frac{g\left(t_{k}^{*}-f_{k}, D_{k}\right) V\left(r_{k}\right)}{\bar{\alpha}}\left(e^{-\bar{\alpha}\left(t_{k}^{*}-f_{k}\right)}-1\right) .
\end{aligned}
$$

Since the hypotheses of lemma 4.1 are satisfied, $V\left(f_{k}\right) \leq$ $(1+\rho \Delta) V\left(r_{k}\right)$ holds. Applying this and equation 29 to the inequality above, we have

$$
\begin{aligned}
& p\left(t_{k}^{*}-f_{k}\right) \triangleq 1+\rho \Delta-\delta \bar{\alpha}\left(t_{k}^{*}-f_{k}\right) \\
& \leq(1+\rho \Delta) e^{-\bar{\alpha}\left(t_{k}^{*}-f_{k}\right)}-\frac{g\left(t_{k}^{*}-f_{k}, \Delta\right)}{\bar{\alpha}}\left(e^{-\bar{\alpha}\left(t_{k}^{*}-f_{k}\right)}-1\right) \\
& \triangleq q\left(t_{k}^{*}-f_{k}\right)
\end{aligned}
$$

Notice that $p(0)=q(0)$. Since $\Delta<\Delta_{3}$ implies $\dot{p}(0)>$ $\dot{q}(0)$, by lemma 3.1 , we know $t_{k}^{*}-f_{k} \geq \xi(\Delta)>0$.

Theorem 4.3: For the sampled-data system in equation 8 , let assumption 2.1 hold. If $r_{0}=f_{0}=0$ and for any $k \in \mathbb{Z}^{+}$,

1) $D_{k}<\Delta=\min \left\{\Delta_{1}, \Delta_{2}, \Delta_{3}, \Delta_{4}\right\}$ holds, where $\Delta_{1}, \Delta_{2}, \Delta_{3} \in \mathbb{R}^{+}$are defined in equation 22,23 and 
28, respectively, $\Delta_{4} \in \mathbb{R}^{+}$is the smallest positive solution to

$$
\rho \Delta_{4}-\xi\left(\Delta_{4}\right) \delta \bar{\alpha}=0
$$

or $\infty$ if the positive solution to equation 35 does not exist, $\xi$ is defined in equation $30, \rho>\bar{\beta} L_{1}(1+\lambda)$, $\delta \in(0,1)$, and $\lambda \in(1, \infty)$.

2) $r_{k+1}$ is triggered by the violation of

$$
\left(E_{1} \bigwedge E_{2}\right) \bigvee E_{3}
$$

where

$$
\begin{aligned}
& E_{1}: V(t) \leq\left(1+\rho \Delta-\delta \bar{\alpha}\left(t-f_{k}\right)\right) V\left(r_{k}\right) \\
& E_{2}: \lambda V(t)>V\left(r_{k}\right) \\
& E_{3}: r_{k} \leq t \leq f_{k}
\end{aligned}
$$

then the sampled-data system 8 is asymptotically stable.

Proof: By lemma 4.1, we know $E_{1}$ and $E_{2}$ always hold when $t=f_{k}$. It is easy to show that $\xi(0)>0$ according to equation 30. Therefore, $\rho D_{k}-\xi\left(D_{k}\right) \delta \bar{\alpha}<0$ holds when $D_{k}=0$. By lemma 3.1 and the definition of $\Delta_{4}$, we have $\rho D_{k}-\xi\left(D_{k}\right) \delta \bar{\alpha}<0$ for all $D_{k}<\Delta_{4}$. Consequently, there must be a positive constant $\epsilon$ such that

$$
\rho \Delta-\xi(\Delta) \delta \bar{\alpha}<-\epsilon,
$$

holds since $\Delta<\Delta_{4}$. We construct a piecewise continuous function $h: \mathbb{R}^{+} \times \mathbb{R}^{n} \rightarrow \mathbb{R}^{+}$in the following way:

$$
h\left(t \mid x_{0}\right)=\left\{\begin{array}{l}
{\left[1+\rho \Delta-\delta \bar{\alpha}\left(t-f_{k}\right)\right] V\left(r_{k}\right), t \in\left[f_{k}, r_{k+1}\right)} \\
(1+\rho \Delta) V\left(r_{k+1}\right), \quad t \in\left[r_{k+1}, f_{k+1}\right)
\end{array}\right.
$$

Because $r_{k+1}$ is triggered by the violation of $E_{1}$ or $E_{2}$, it is easy to show $V(t) \leq h\left(t \mid x_{0}\right)$ for all $t \in\left[f_{k}, r_{k+1}\right)$ and $k \in \mathbb{Z}^{+}$. Since $D_{k}<\Delta \leq \Delta_{1}$ holds, by lemma 4.1 , $V(t) \leq(1+\rho \Delta) V\left(r_{k}\right)=h\left(t \mid x_{0}\right)$ holds for all $t \in\left[r_{k}, f_{k}\right)$ and $k \in \mathbb{Z}^{+}$. Therefore,

$$
V(t) \leq h\left(t \mid x_{0}\right)
$$

holds for all $t \in \mathbb{R}^{+}$. We now show that $\lim _{t \rightarrow \infty} h\left(t \mid x_{0}\right)=$ 0 . Two cases are considered.

Case I: $r_{k+1}$ is triggered by the violation of $E_{1}$. Then

$$
\begin{aligned}
& h\left(f_{k} \mid x_{0}\right)=(1+\rho \Delta) V\left(r_{k}\right) \\
& h\left(f_{k+1} \mid x_{0}\right)=(1+\rho \Delta) V\left(r_{k+1}\right)
\end{aligned}
$$

Since $r_{k+1}$ is triggered by the violation of $E_{1}$, we have

$$
V\left(r_{k+1}\right)=\left[1+\rho \Delta-\delta \bar{\alpha}\left(r_{k+1}-f_{k}\right)\right] V\left(r_{k}\right) .
$$

By lemma 4.2, we know $r_{k+1}-f_{k} \geq \xi(\Delta)$. So

$$
V\left(r_{k+1}\right) \leq[1+\rho \Delta-\delta \bar{\alpha} \xi(\Delta)] V\left(r_{k}\right)
$$

holds. Combining this inequality with equation 43 , we have $h\left(f_{k+1} \mid x_{0}\right) \leq[1+\rho \Delta-\delta \bar{\alpha} \xi(\Delta)] h\left(f_{k} \mid x_{0}\right)$, which implies

$$
h\left(f_{k+1} \mid x_{0}\right) \leq(1-\epsilon) h\left(f_{k} \mid x_{0}\right)
$$

according to equation 40 .
Case II: $r_{k+1}$ is triggered by the violation of $E_{2}$. Following the similar analysis for case I, we have

$$
h\left(f_{k+1} \mid x_{0}\right)=\frac{1}{\lambda} h\left(f_{k} \mid x_{0}\right) .
$$

Equation 44 and 45 implies

$$
\lim _{k \rightarrow \infty} h\left(f_{k} \mid x_{0}\right)=0
$$

since $\lambda \in(1, \infty)$. Notice that $h\left(t \mid x_{0}\right) \leq h\left(f_{k} \mid x_{0}\right)$ holds for all $t \geq f_{k}$. So equation 46 implies

$$
\lim _{t \rightarrow \infty} h\left(t \mid x_{0}\right)=0 \text {. }
$$

Equation 42 and 47 are sufficient to conclude that the sampled-data system is asymptotically stable.

Remark 4.1: Event $E_{2}$ in equation 38 is used to control the distance between $V\left(r_{k+1}\right)$ and $V\left(r_{k}\right)$. The reason to do this is that if $V\left(r_{k+1}\right)$ is arbitrarily small, the deadline will go to zero, although the sample period might be enlarged. There is a tradeoff between periods and the predicted deadlines.

\section{Simulations}

In this section, we used the inverted pendulum problem in [13] to demonstrate the proposed event-triggered scheme. The plant's linearized state equations were

$$
\begin{aligned}
\dot{x} & =\left[\begin{array}{cccc}
0 & 1 & 0 & 0 \\
0 & 0 & -m g / M & 0 \\
0 & 0 & 0 & 1 \\
0 & 0 & g / \ell & 0
\end{array}\right] x+\left[\begin{array}{c}
0 \\
1 / M \\
0 \\
-1 /(M \ell)
\end{array}\right] u \\
& =A x+B u
\end{aligned}
$$

where $M=10, m=1, \ell=3$, and $g=10$. The system's initial state was the vector $x_{0}=\left[\begin{array}{llll}0.98 & 0 & 0.2 & 0\end{array}\right]^{T}$. The controller is $u=K x$, where $K=\left[\begin{array}{llll}2 & 12 & 378 & 210\end{array}\right]$. The Lyapunov function we used for the continuous closedloop system is $V(x)=\sqrt{x^{T} P x}$, where

$$
P=\left(\begin{array}{cccc}
7 & 21 & 222 & 127 \\
21 & 106 & 1180 & 675 \\
222 & 1180 & 26578 & 14873 \\
127 & 675 & 14873 & 8327
\end{array}\right)
$$

We compared our event-triggering scheme with the eventtriggering scheme in [9], the self-triggering scheme in [13], and MATI in [6] when $D_{k}=0$. Recall that the eventtriggering scheme in [9] samples the state when

$$
e_{k}^{T}(t) P e_{k}(t)=p^{2} x^{T}(t) P x(t) .
$$

where $p$ is the real constant

$p=\frac{\sigma_{\min }(P)}{2 \sigma_{\max }(P)} \frac{\sigma_{\min }\left(-P(A+B K)-(A+B K)^{T} P\right)}{\|P B K\|}$.

When $D_{k}=0$, the self-triggering scheme in [13] triggers the $k+1$ th task release, $r_{k+1}$, in the following way:

$$
r_{k+1}=r_{k}+\frac{1}{\bar{a}} \ln \left(1+\frac{\bar{a}\left\|\sqrt{N} x\left(r_{k}\right)\right\|}{\left\|\sqrt{M}(A+B K) x\left(r_{k}\right)\right\|}\right)
$$

where $M=\frac{3}{4} I+P B B^{T} P$ and $N=\frac{3}{8} I+P B B^{T} P$ and $\bar{a}=\left\|\sqrt{M} A \sqrt{M}^{-1}\right\|$. 
MATI in [6] is defined by

$$
\begin{cases}\frac{1}{L r} \arctan \frac{r(1-\bar{\lambda})}{2 \frac{\lambda}{1+\lambda}\left(\frac{\bar{\gamma}}{L}-1\right)+1+\bar{\lambda}} & \bar{\gamma}>\bar{L} \\ \frac{1-\bar{\lambda}}{L(1+\lambda)} & \bar{\gamma}=\bar{L} \\ \frac{1}{L r} \operatorname{arctanh} \frac{r(1-\bar{\lambda})}{2 \frac{\lambda}{1+\lambda}\left(\frac{\bar{\gamma}}{L}-1\right)+1+\bar{\lambda}} & \bar{\gamma}<\bar{L}\end{cases}
$$

where $\bar{\lambda}=0, \bar{L}=\max \left(0.5 \sigma_{\max }\left(-B K-K^{T} B^{T}\right), 0\right), \bar{\gamma}$ is the $\mathcal{L}_{2}$ gain for the closed-loop system $\left(\dot{x}=A_{c l} x+B K e\right)$ from $e$ to $-A_{c l} x$, and $r=\sqrt{\left|\frac{\bar{\gamma}^{2}}{L^{2}}-1\right|}$.

The average periods generated by different schemes are listed in Table I. It is obvious that our event-triggered scheme has a much longer average sample period.

TABLE I

COMPARISON OF DIFFERENT SCHEMES

\begin{tabular}{|l|c|}
\hline Schemes & Average Periods \\
\hline \hline Our event-triggering scheme $(\delta=0.2)$ & 0.4816 \\
\hline Event-triggering scheme in [9] & $<10^{-5}$ \\
\hline Self-triggering Scheme in [13] & 0.1782 \\
\hline MATI in [6] & 0.0169 \\
\hline
\end{tabular}

We then took a look at non-zero delay cases. The parameters were computed based on equation 7 in remark 2.2, where $\bar{\alpha}=0.015, \beta=\bar{\beta}=1042.8, L=45.58$, $L_{1}=1.91, \rho=5993, \bar{\lambda}=2, \delta=0.8$. The deadline based on theorem 4.3 is around $10^{-12}$. This deadline is extremely small, although it is at the same level of the predicted deadline in [9] which is around $10^{-13}$. It is because the large condition number of $P$ leads to a small $\bar{\alpha}$ and a big $\bar{\beta}$, which directly affect the solutions to equation 28 and 35 . Notice that the method we proposed is only for showing the existence of non-zero deadlines. In practice, for systems with a large condition number of $P$, it is better to use dynamic deadlines because of its "on-line" nature.

We then added random delays satisfying $D_{k} \leq 0.1$ into the proposed event-triggered feedback system to see how robust this system can be to delays. We used the violation of equation 36 to trigger the next release with $\Delta=0$. The results are presented in figure 2. The state trajectories are shown in the top plot of figure 2. From this plot, we can see that, the event-triggered feedback system still converges to the equilibrium even when $D_{k}$ can be as large as 0.1 . The bottom plot of figure 2 provides the sample periods in this system. The average period is 0.1882 , which is definitely larger than the periods offered by the prior work. These simulation results suggest that the event-triggered feedback system is robust to delays. How to obtain a tighter estimate of the deadline would be an interesting future topic.

\section{CONCLUSIONS}

This paper proposed a new event-triggering scheme. The resulting event-triggered feedback systems are guaranteed to be asymptotically stable, provided that the continuous systems are stabilizable. We show that the task periods and deadlines generated by our scheme are bounded strictly away from zero if the continuous systems are ISS with respect
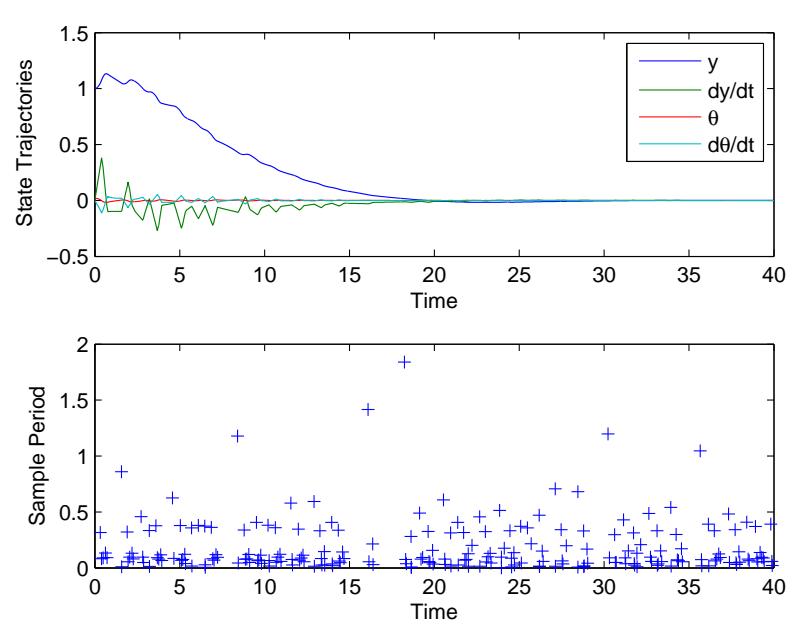

Fig. 2. An event-triggered feedback system with $D_{k} \leq 0.1$

to measurement errors. Simulation results indicate that our event-triggered scheme has a much larger average period than the previous event/self-triggered schemes. Moreover, our scheme also appears to be robust to task delays.

\section{REFERENCES}

[1] Y. Zheng, D. Owens, and S. Billings, "Fast sampling and stability of nonlinear sampled-data systems: Part 2. sampling rate estimations," IMA Journal of Mathematical Control and Information, vol. 7, pp. 13-33, 1990.

[2] D. Nesic, A. Teel, and E. Sontag, "Formulas relating $\mathcal{K} \mathcal{L}$ stability estimates of discrete-time and sampled-data nonlinear systems," Systems and Control Letters, vol. 38, pp. 49-60, 1999.

[3] L. Zaccarian, A. Teel, and D. Nesic, "On finite gain $\mathcal{L}_{p}$ stability of nonlinear sampled-data systems," System and Control Letters, vol. 49, pp. 201-212, 2003.

[4] G. Walsh, H. Ye, and L. Bushnell, "Stability analysis of networked control systems," IEEE Transactions on Control Systems Technology, vol. 10, no. 3, pp. 438-446, 2002.

[5] D. Nesic and A. Teel, "Input-output stability properties of networked control systems," IEEE Transactions on Automatic Control, vol. 49, pp. 1650-1667, 2004.

[6] D. Carnevale, A. R. Teel, and D. Nesic, "Further results on stability of networked control systems: a lyapunov approach," IEEE Transactions on Automatic Control, vol. 52, pp. 892-897, 2007.

[7] K. Arzen, "A simple event-based PID controller," in Proceedings of the 14th IFAC World Congress, 1999.

[8] K. Astrom and B. Bernhardsson, "Comparison of Riemann and Lebesgue sampling for first order stochastic systems," in Proceedings of IEEE Conference on Decision and Control, 1999.

[9] P. Tabuada and X. Wang, "Preliminary results on state-triggered scheduling of stabilizing control tasks," in Proceedings of IEEE Conference on Decision and Control, 2006.

[10] P. Tabuada, "Event-Triggered Real-Time Scheduling of Stabilizing Control Tasks," Automatic Control, IEEE Transactions on, vol. 52, no. 9, pp. 1680-1685, 2007.

[11] M. Velasco, P. Marti, and J. Fuertes, "The self triggered task model for real-time control systems," in Work-in-Progress Session of the 24th IEEE Real-Time Systems Symposium (RTSS03), 2003.

[12] M. Lemmon, T. Chantem, X. Hu, and M. Zyskowski, "On selftriggered full information h-infinity controllers," in Hybrid Systems: computation and control, 2007.

[13] X. Wang and M. Lemmon, "Self-triggered feedback control systems with finite-gain $\mathcal{L}_{2}$ stability," accepted to IEEE Transactions on Automatic Control.

[14] H. Ye, A. Michel, and L. Hou, "Stability theory for hybrid dynamical systems," Automatic Control, IEEE Transactions on, vol. 43, no. 4, pp. 461-474, 1998. 